\title{
Evaluation of Conventional and Molecular Techniques in the Diagnosis of Fungal Rhinosinusitis
}

\author{
Authors \\ Nitya Verma ${ }^{1}$, Prashant Gupta ${ }^{2}$, Veerendra Verma ${ }^{3}$, \\ Dakshina Bisht $^{1}$, S. P.Agarwal ${ }^{3}$, Ajay Kumar Singh ${ }^{4}$, Abrar Ahmad ${ }^{2}$ \\ ${ }^{1}$ Department of Microbiology, Santosh Medical University, Ghaziabad, NCR, Delhi \\ ${ }^{2}$ Department of Microbiology, King George's Medical University, Lucknow \\ ${ }^{3}$ Department of ENT, King George's Medical University, Lucknow \\ ${ }^{4}$ Department of Pathology, King George's Medical University, Lucknow
}

Introduction: Fungal infection of the nose and paranasal sinuses is increasing in both immunocompetent and immunocompromised patients. Limited studies on spectrum of fungi causing fungal rhinosinusitis (FRS) and the types of FRS are scarce from this region of India. We therefore analyzed the suspected cases of FRS by clinical, radiological, histopathological, mycological and molecular methods and categorized them in to non-invasive and invasive forms.

Objective: To evaluate laboratory methods in the diagnosis of fungal rhinosinusitis and to find out the spectrum of fungi causing various forms of FRS

Methods: 44 patients of chronic rhinosinusitis were enrolled on the basis of history, clinical examination, and radiological findings from the E.N.T. department after obtaining their consent. Functional endoscopic sinus surgery was performed and tissues were examined histopathologically, by fungal culture and PCR. Antimicrobial susceptibility testing of the fungal isolates were done by disk diffusion (M51-A) \& broth micro dilution (M38-A2) methods of CLSI.

Result: Out of 44 clinically and radiologically suspected patients of chronic rhinosinusits, 23 (52\%) were positive for fungal rhinosinuistis. In 44 cases, 18 (40.9\%) cases were PCR positive, 12 (27\%) were culture positive, 8(18\%) were positive by $\mathrm{KOH}$ microscopy and 4 (9\%) were positive on histopathology. Aspergillus flavus was found to be the most common fungal isolate causing fungal rhinosinusitis

Conclusion: We found laboratory methods are essential in confirming the diagnosis of FRS. Among all the laboratory methods, though culture is an important diagnostic tool, PCR were found to be more significant than other methods.

\section{Introduction}

Rhinosinusitis is an inflammation of the nasal and paranasal sinus. It is defined chronic when it lasts longer than 3 months without complete symptoms resolution. Fungal rhinosinusitis can be invasive and non-invasive. ${ }^{(1)}$
Invasive FRS is subcategorized into acute invasive, chronic invasive and chronic granulamatous forms. Non-invasive FRS is subcategorized into localized fungal colonization, Allergic fungal rhinosinusitis (AFRS) and fungal ball. ${ }^{(2)}$

Most of the fungi causing sinusitis are common saprobes. Although Aspergillus species are the 
major aetiological agents, the fungi like Schizophyllum commune, Alternaria, Curvularia and Bipolaris are also reported to cause sinusitis. The development and progression of the disease depends upon several factors like immunological status, site, duration and presence of atopy. ${ }^{(3)}$

Currently diagnosis of fungal rhinosinusitis depends on radiological, histopathological examination, $\mathrm{KOH}$ microscopy and culture from nasal tissue. Conventional culture based phenotypic identification techniques often include significant delays and can fail to yield growth in tissue samples. Rapid diagnosis from surgical tissues is often needed in acute invasive infections. In addition, histopathology observations of fungal shape and arrangement may not be sufficient for the accurate identification of fungal species if only a limited quantity of anamorphic fungal hyphae is present. Therefore, to improve the outcome for fungal rhinosinusitis patients, the rapid and accurate identification and detection of pathogenic fungal species.

Our complete strategy consists of a rapid universal DNA extraction followed by PCR amplification with universal primers for $28 \mathrm{~S}$ rDNA and compare with conventional methods ${ }^{(4)}$

We analyzed the suspected cases of FRS by clinical, radiological, histopathological, mycological and molecular methods and categorized them into non-invasive and invasive forms. We developed an assay combining PCR amplification and Microscopy to detect pathogens in tissue specimens.

\section{Material and Methods}

44 patients of chronic rhinosinusitis were enrolled on the basis of history, clinical examination, and radiological findings from the ENT department after obtaining their consent. Functional endoscopic sinus surgery was performed and tissue were examined by histopathologically, fungal culture and PCR. Antimicrobial susceptibility testing of the fungal isolates were done by disc diffusion (M51-A) and broth micro dilution (M38-A2) methods of CLSI.

\section{Inclusion criteria}

Individual having either at least two major or one major and two minor criteria had considered for inclusion. ${ }^{(5)}$
Patients who had symptoms and sign for three months of chronic sinusitis patients.

\section{Exclusion criteria}

Patients suffering from other diseases like congenital muco-cillary disorder, Atrophic rhinitis, Systemic disease causing problems.

\section{Culture and Microscopy:}

The samples were examined directly in to $20 \%$ $\mathrm{KOH}$ mount. Culture was done on Sabourad's dextrose agar (SDA) with chlormphenicol and incubated at $25{ }^{0} \mathrm{C}$ and $37{ }^{\circ} \mathrm{C}$ respectively. The cultures were examined from 5 to 21 days regularly and identified systematically. All histological samples were stained with haemotoxylin and eosin and with periodic acid Schiff staining. If sample was negative for fungi then the gomori methamine silver staining method was done.

\section{DNA extraction}

Tissue sections were cut with a microtome. Before the first cut and after each sample, the microtome and other instruments were cleaned with cleaning benzine followed by $2 \mathrm{M} \mathrm{HCl}$ and rinsed with sterile water. One tissue section ( $5 \mu \mathrm{m}$ thick) or a loopful of fresh tissue was suspended in column, and DNA extraction was performed according to the Kit (ZR fungal/bacterial mini prep by Zymo research).

\section{PCR}

Universal primers for the 28S rDNA originally described by Sandhu et al.(4) were used to amplify a DNA sequence $260 \mathrm{bp}$ in length (primers U1 [5'-GTG AAA TTG TTG AAA GGG AA-3'] and U2 [5'-GAC TCC TTG GTC CGT GTT-3']). PCR amplifications were carried out in $50-\mu 1$ reaction volumes. Cycling conditions were as follows: initial denaturation at $94^{\circ} \mathrm{C}$ for $7 \mathrm{~min}$ followed by 35 cycles of denaturation at $94^{\circ} \mathrm{C}$ for $1 \mathrm{~min}$, annealing at $45^{\circ} \mathrm{C}$ for $1 \mathrm{~min}$, and extension at $72^{\circ} \mathrm{C}$ for $1 \mathrm{~min}$ followed by a final extension phase at $72^{\circ} \mathrm{C}$ for $10 \mathrm{~min}$.

To minimize the risk of contamination, a laminar flow hood and aerosol-resistant micropipette tips were used and areas for the preparation of master mix, extraction of DNA, template preparation, setting up of PCR, and post-PCR analysis were physically separated. 
Amplification products were separated by electrophoresis in a 1\% agarose gel by using standard techniques, subsequently stained with ethidium bromide, and analyzed with a gel documentation system (MWG-Biotech). PCR products $260 \mathrm{bp}$ in length were interpreted as evidence of successful target amplification.

\section{Results}

44 patients of chronic rhinosinusitis were enrolled in the E.N.T. department from $4^{\text {th }}$ July 2013 to $3^{\text {rd }}$ July 2014 bases on above said inclusion criteria. All the patients have given their consent after admission in the department.

Maximum number of cases were found to be in age group male and female both 20-29 yrs (28.5 $\%$ ), followed by $30-39$ yrs (26.2\%). We observed that majority $(64 \%)$ of cases were received in winters (October-February) as month of November reported highest number of cases, while rest of the cases (36\%) came from summer (March-August) with September reporting none . Majority of patients $57 \%$ (24) belonged to rural areas while $43 \%$ (18) patients belonged to urban areas. The most common clinical presentation in case of chronic sinusitis were nasal obstruction $(92.8 \%)$ followed by headache $(81 \%)$, nasal discharge $(73.8 \%)$ and facial congestion $(33.3 \%)$. Twenty one $(50 \%)$ cases were confirmed as positive by any of the method used in laboratory diagnosis. In 42 cases, 16 (38\%) cases were PCR positive. Out of these 16 , ten were also culture positive. $40 \%$ cases were found normal on histopathological findings, in remaining $60 \%$ cases, inflammatory polyp was the most common finding followed by malignancy (12\%).

Out of 12 culture positive cases Aspergillus flavus $(91.66 \%)$ was found to be most common fungi followed by one case of Schizophyllum commune $(8.33 \%)$ causing FRS. All the strains were within the ECV range $100 \%$ respectively with Itraconazole, Voriconazole, Amphotericine B, and Caspofungin.

Most consistent radiological findings in clinically suspected cases of chronic sinusitis were sinus expansion followed by hyper-attenuation and mucosal hypertrophy.

Table 1: Classification of fungal rhinosinusitis according to Histopathology

\begin{tabular}{|l|c|}
\hline Fungal Rhinosinusitis & Number Patients $(\mathbf{n = 4 )}$ \\
\hline Acute Invasive & 0 \\
\hline Chronic invasive & 1 \\
\hline Chronic granulomatous & 1 \\
\hline AFRS & 2 \\
\hline Fungal ball & 0 \\
\hline
\end{tabular}

Table 2: Distribution of cases of chronic sinusitis according to age and sex

\begin{tabular}{|l|c|c|c|c|c|c|}
\hline \multirow{2}{*}{$\begin{array}{l}\text { Age } \\
\text { Group/years) }\end{array}$} & \multicolumn{2}{|c|}{ Male } & \multicolumn{2}{c|}{ Female } & \multicolumn{2}{c|}{ Total } \\
\cline { 2 - 7 } & No & $\%$ & No & $\%$ & No & $\%$ \\
\hline $10-19$ & 4 & 17.4 & 2 & 10.5 & 6 & 14.3 \\
\hline $20-29$ & 6 & 26.1 & 6 & 31.7 & 12 & 28.5 \\
\hline $30-39$ & 8 & 33.4 & 4 & 21 & 12 & 27.2 \\
\hline $40-49$ & 2 & 8.7 & 5 & 25 & 7 & 16 \\
\hline $50-59$ & 3 & 13 & 2 & 10.5 & 5 & 12 \\
\hline$>60$ & 1 & 4.4 & 1 & 5.3 & 2 & 4.7 \\
\hline Total & 24 & 100 & 20 & 100 & 44 & 100 \\
\hline
\end{tabular}


Table: 3 Distribution According to the direct microscopy, culture, PCR, and Histopathology in patients of chronic fungal rhinosinusitis

\begin{tabular}{|l|c|c|c|c|c|}
\hline $\begin{array}{l}\text { Results } \\
(\mathbf{n}=\mathbf{4 4})\end{array}$ & $\begin{array}{c}\text { Direct } \\
\text { microscopy }\end{array}$ & Culture & PCR & Radiology & Histopathology \\
\hline Positive & 8 & 12 & 18 & 6 & 4 \\
\hline Negative & 36 & 32 & 26 & 38 & 40 \\
\hline
\end{tabular}

Table 4: Diagnostic evolution of various diagnostic methods

\begin{tabular}{|l|c|c|c|c|}
\hline $\begin{array}{l}\text { Diagnostic } \\
\text { methods }\end{array}$ & Sensitivity & Specificity & $\begin{array}{c}\text { Positive predictive } \\
\text { value (\%) }\end{array}$ & $\begin{array}{c}\text { Negative predictive } \\
\text { value (\%) }\end{array}$ \\
\hline PCR & 75 & 71.88 & 50 & 88.46 \\
\hline Microscopy & 50 & 93.75 & 75 & 83.33 \\
\hline Radiology & 27.7 & 90.91 & 50 & 78.95 \\
\hline Histopathology & 25 & 100 & 100 & 78.05 \\
\hline
\end{tabular}

Table 5: Combine evaluation of microscopy and PCR with gold standard (culture)

\begin{tabular}{|l|c|c|c|c|}
\hline Diagnostic methods & Sensitivity (\%) & Specificity (\%) & $\begin{array}{c}\text { Positive predictive } \\
\text { value (\%) }\end{array}$ & $\begin{array}{c}\text { Negative predictive } \\
\text { value (\%) }\end{array}$ \\
\hline PCR + Microscopy & 81.82 & 81.82 & 60 & 93.10 \\
\hline
\end{tabular}

\section{Discussion}

The aim of present study was to evaluate the conventional and molecular methods for the diagnosis of fungal rhino-sinusitis. The purpose of the study was to evaluate and established PCR amplification method as a diagnostic tool for the identification of fungi in tissue specimens obtained from chronic rhinosinuitis patients. To our knowledge no study based on molecular techniques provides epidemiological data on fungi causing FRS from India. Molecular detection is a sensitive diagnostic tool for the detection of pathogen which is non - viable or dormant due to the use of antifungal therapy and other unfavorable condition. We targeted $28 \mathrm{~S}$ rDNA sequences due to its large size reveals adequate species specific differentiation to distinguish closely related organism, it is a highly conserved region of fungal genome sequences ${ }^{(4)}$.

Generally sinusitis affects the patients' general health, vitality and its social well beings. The diagnosis of sinusitis was established by examination of sinus tissues obtained during surgery. In the present study the incidence of FRS was $52.2 \%$ among patients with rhinosinusitis based on direct microscopy, culture, histopathology findings as well as molecular direction. Earlier studies have reported rates of
FRS from $7.3 \%$ to $25 \%$ but we have good rate of incidence of FRS in Indian scenario ${ }^{(6)}$. Patients with CRS presented with following clinical sign and symptoms $96 \%$ (patients) were complaining about nasal obstruction, where as $88.16 \%(67 \%)$ having nasal discharge followed by purulence of nasal cavity ,facial congestion and other complaints. We found that majority of cases were received in the months of November $(18.42 \%)$ and December $(14.47 \%)$ out of 44 patients of CRS. Maximum number of patients $57.89 \%$ belongs to urban areas while $42.10 \%$ belongs to rural areas. ${ }^{(7)}$ We have evaluated the molecular identification of fungus over conventional method. Positivity of PCR shows that it is most reliable and least time consuming diagnosis process for the identification of FRS. Out of 44 patients $8(18.1 \%)$ were found to be positive by direct microscopy, $12(27.3 \%)$ patients positive by culture, $18(40.9 \%)$ were found to be PCR positive, only 4 (9\%) positive by Histopathology. The ratio of male versus female patients in our study suffering from Fugal rhinosinusitis were 1.2:1, which is in agreement with study done by Das et al. 2009 , similar results were found by Michael et al in the year 2008. The mean age was 33.1years and they ranged from 9 to 74 years $^{(8)(9)}$. 
The result of our PCR assay detects the fungal DNA in 18 cases of FRS out of 44 cases of CRS. Whereas only 12 patients were found to be by culture. This result indicates that the detection of fungal DNA by PCR in case of CRS is superior as compared to culture. The sensitivity (75\%) and NPV $(88.46 \%)$ as well as nearby perfect specificity of PCR shows that it is promising procedure for the diagnosis of FRS. The NPV of PCR are the best among the entire test, it also indicates that PCR is the best method among all to rule out the disease.

In the present study $40 \%$ of our subjects were found to be PCR positive which in agreement with study done by Mohammed et al. ${ }^{(10)}$ PCR and microscopy in combination proved to be best method to diagnose FRS with $81.82 \%$ (sensitivity), $81.82 \%$ specificity and $93.10 \%$ NPV.

\section{Conclusion}

In the present studies we have analysed fresh tissue sample by conventional as well as molecular method. The overall results suggest that molecular method is accountable for FESS tissue samples. It is also suggested that molecular diagnostic method for FRS in clinical practice should be taken in consideration for timely management of patients. Diagnosis should not be only on the basis of clinic -radiological findings. Among all the test PCR and Microscopy in combination proved to be the best method to diagnose FRS.

\section{References}

1. Patron Piromchai, Pornthep kasemsiri, Supawan Laohasiriwong, Sanguansak Thanaviratananich. Chronic rhinosinusitis and emerging treatment options. International journal of general medicine, 2013; 6: 453-464.

2. Zachary M. Soler and Rodney J. Schlosser. The role of fungi in disease of the nose and sinuses. Am J Rhinol \& Allergy, 2012; 26: 351-358.

3. Schubertz MS. Allergic fungal sinusitis pathophysiology, diagnosis and management. Med Mycol, 2009; 47:S32430.

4. Sandhu G, Kline BC, Atockman L. Molecular probes for diagnosis of fungal infections. J Clin Microbiol 1995; 33:2913-19.

5. Lanza D, Kennedy DW. Adult rhinosinusitis defined. Otolaryngol Head Neck Surg 1997; 117(3 pt 2):S1-7

6. Badiee P, Gandomi B, Sabz G, Khodami B, Choopanizadeh $M$, Jafarian $H$. Evaluation of nested PCR in diagnosis of fungal rhinosinusitis. Iran J Micro, 2015; 7(1):62-66.

7. Prateek S, Banerjee G, Gupta P, Singh M, Goel M, Verma V. Fungal rhinosinusitis: A prospective study in a university hospital of Uttar Pradesh. Ind Jour Med Micro, 2013; 31(1): 266-269.

8. Das A , Bal A, Chakrabarti, Panda N, Joshi K. Spectrum of fungal rhinosinusitis; histopathologist's perspective. Wiley on line jour, 2009; 64(7):854-859.

9. Michael RC, Michael JS, Ashbee RH, Mathews MS. Mycological profile of fungal sinusitis: An audit of specimens over a 7 year period in a tertiary care hospital in Tamil nadu. Indian J Pathol Microbio, 2008; 51: 493-496.

10. Mohammaed M, Naggar EI, El-morsy S, Abou Beith A, and Khafagi Y. Allergic fungal sinusitis: Detection of Universal fungal, Aspergillus and Bipolaris DNA in sinus aspirate using polymerase chain reaction and evaluation of Aspergillus specific IgE. Egypt. Journ of Med. 